\title{
INNOVACIÓN EN LA ORIENTACIÓN UNIVERSITARIA. UNA EXPERIENCIA: \\ RED DE ESTUDIANTES MENTORES EN LA UNIVERSIDAD COMPLUTENSE (REMUC)
}

\author{
Laura Oliveros Martín-Varés \\ Mercedes García García \\ Covadonga Ruiz de Miguel \\ Andrés Valverde Macías \\ Universidad Complutense de Madrid
}

RESUMEN: En este artículo se presenta una experiencia de mentoría en la universidad que utiliza como herramienta esencial un portal web diseñado ad hoc.

Siguiendo la iniciativa surgida en el Departamento de Didáctica y Organización Escolar y Métodos de Investigación y Diagnóstico en Educación de la Facultad de Educación de Sevilla en el curso 2001-2002, un grupo de profesores pertenecientes al Departamento MIDE de la Facultad de Educación de la UCM, adaptó e implantó la experiencia en dicha Universidad.

La descripción de la Red de Estudiantes Mentores en la Universidad Complutense (REMUC) incluye: cómo surge la idea del proyecto, sus objetivos, las fases que fueron necesarias para hacerlo realidad, la estructura y elementos que lo componen, el modelo de intervención en que se sustenta, y las herramientas que utiliza. Así mismo se incluyen los resultados obtenidos durante el desarrollo, su evaluación y las conclusiones derivadas de la experiencia.

ABSTRACT: In this work a mentoring experience at the university level is presented. The initiative, arised at the Department DOE-MIDE of the Faculty of Education of Seville in the course 2001-2002, has been adapted and implanted at the University Complutense of Madrid by a group of professors belonging to the Department of Methods of Investigation and Diagnosis in Education of the Faculty of Education.

The description of the Net of Students Mentors in the University Complutense of Madrid (REMUC) is presented and includes: how the idea arised, the objectives, the phases that were necessary to make it reality, the structure and elements that composed it, the intervention pattern that sustains it, 
and the tools used. The development of the followed process is also described as well a as the evaluation and the experience's conclusions.

PALABRAS CLAVE: Innovación en la universidad, orientación universitaria, mentoría y ayuda entre iguales, necesidades de los estudiantes universitarios.

KEYWORDS: Innovation at university, university guidance, peer mentoring, university student's needs.

\section{INTRODUCCIÓN}

La Red de Estudiantes Mentores en la Universidad Complutense (REMUC), es una experiencia que tiene su origen en el Proyecto SIMUS (Sistema de Mentores en la Universidad de Sevilla) realizado en dicha ciudad en el curso académico 2001-2002, avalado como proyecto de investigación por el Instituto de Ciencias de la Educación (ICE) de la Universidad de Sevilla. Los resultados allí alcanzados llevaron a los promotores de la idea a extenderla a otras universidades. Así, en el curso académico 2002-03 se inicia la experiencia en Madrid, integrada en un proyecto más amplio, coordinado y gestionado desde la Facultad de Educación de la Universidad de Sevilla respaldado, en Sevilla por el grupo de investigación MIDO (HUM 107 del Plan Andaluz de Investigación), y en Madrid, por el Proyecto de Innovación Educativa UCM, convocatoria 2003.

El marco teórico que justifica la necesidad de este proyecto ya ha sido presentado en un artículo anterior (Valverde Macias, Ruiz de Miguel, García Jiménez y Romero Rodríguez, 2003) al que desde aquí remitimos. Lo que ahora se presenta es el desarrollo de la experiencia en la Universidad Complutense de Madrid en la que, por sus propias características (macro-contexto un tanto despersonalizado), cobran aún más sentido los objetivos planteados inicialmente en la Universidad de Sevilla.

\section{DESCRIPCIÓN DE LA RED DE ESTUDIANTES MENTORES EN LA UNIVERSIDAD COMPLUTENSE (REMUC)}

La Red de Estudiantes Mentores en la Universidad Complutense es un sistema de orientación para el alumnado de nuevo ingreso con el fin de facilitarles su tránsito desde la Educación Secundaria a la Educación Superior. Aporta, en comparación con otros proyectos que persiguen el mismo fin, el hecho de que la relación de ayuda ofrecida a los alumnos de primer curso la efectúan otros alumnos de últimos cursos de la misma titulación, que ejercen de mentores. Más concretamente, la Red de Estudiantes Mentores en la Universidad Complutense consiste en un programa de orientación universitaria entre iguales para que los alumnos de últimos cursos de carrera (mentores) desarrollen tareas de tutorización con los alumnos de primero de su titulación (mentorizados) con el fin de facilitarles su incorporación a la Educación Universitaria. Con ello se consigue, en términos cuantitativos, una mayor cobertura de la atención y ayuda dada a los alumnos de primero y, desde una perspectiva cualitativa, una mayor empatía entre quienes ejercen la relación de ayuda y quienes la reciben. Como valor añadido verificado en ésta, como en anteriores experiencias de mentoría y de tutoría entre iguales (Leyva y Oliveros, 1996), también se logran beneficios en los propios mentores. 
Para poder desarrollar esta función, los alumnos mentores fueron previamente formados y orientados por un grupo de profesores del Departamento MIDE de la Facultad de Educación.

Administrativamente, el proceso ha sido posible gracias a la inclusión de una asignatura genérica, dentro de la optatividad que la Universidad Complutense ofrece a sus alumnos. Esta asignatura, como genérica que es, pueden cursarla alumnos de cualquiera de las distintas titulaciones que se imparten en la Universidad Complutense. El único requisito impuesto, indispensable por la misma naturaleza de la experiencia, era que sólo podían matricularse alumnos de últimos cursos. En el caso de las Diplomaturas deberían ser de tercero y en el de las Licenciaturas, indistintamente de cuarto o quinto.

Esta materia, denominada Red de estudiantes mentores en la Universidad, es una asignatura anual de 9 créditos, de los cuales 1,5 son teóricos, desarrollados a través de clases presenciales impartidas a los alumnos matriculados a fin de formarles como mentores, y los 7,5 restantes créditos son de carácter práctico dedicados a la labor de mentorización que éstos alumnos han de ejercer, una vez formados, con los alumnos de primero (alumnos mentorizados).

Aún cuando las clases presenciales ocuparon una pequeña parte del curso académico, la formación y orientación que ofrecieron los profesores tutores continuó a lo largo de todo el proceso bajo el soporte de un campus virtual creado a tal fin.

\section{Objetivos}

El objetivo central de carácter general, tal y como ya se ha expuesto, fue facilitar a los alumnos de primer curso (mentorizados) su incorporación a la vida universitaria. Junto a éste, como objetivo general derivado o de segundo orden, se pretendía también aportar beneficios en conocimientos, actitudes y procedimientos a los alumnos de últimos cursos al ejercer su papel de mentores. Algunos de estos beneficios estaban planificados, es decir, eran objetivos a lograr en el proceso de formación; otros han formado parte del curriculum oculto, esto es, no planificados pero si obtenidos, como después se pondrá de manifiesto en la valoración de la experiencia.

Los objetivos específicos se diferenciaron en función de que fueran dirigidos a los alumnos que se matricularon en la asignatura (mentores), o los que se proyectaron para los alumnos con los que éstos trabajaron (mentorizados). Pueden consultarse cada uno de ellos en el cuadro 1. 


\begin{tabular}{|c|c|c|}
\hline & Para los Mentores & Para los Mentorizados \\
\hline 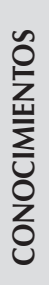 & $\begin{array}{l}\text { Desarrollar competencias técnicas } \\
\text { (saber)* } \\
\text { Conocer los objetivos del proyecto } \\
\text { Conocer el proceso de mentoría }\end{array}$ & $\begin{array}{l}\text { Conocer las técnicas de dinámica de grupos } \\
\text { Conocer los itinerarios formativos de su } \\
\text { titulación, requisitos y exigencias docentes } \\
\text { Conocer las posibilidades que ofrece la } \\
\text { universidad (información sobre becas, } \\
\text { ayudas, etc) } \\
\text { Conocer las posibilidades de formación } \\
\text { externas a la propia universidad }\end{array}$ \\
\hline 岂 & $\begin{array}{l}\text { Desarrollar competencias sociales } \\
\text { y de participación (saber ser) }{ }^{*} \\
\text { Valorar disposición para el trabajo } \\
\text { en grupo } \\
\text { Favorecer la empatía } \\
\text { Potenciar la iniciativa }\end{array}$ & $\begin{array}{l}\text { Adoptar una postura activa frente a su propia } \\
\text { formación } \\
\text { Generar una actitud favorable hacia el } \\
\text { trabajo colaborativo }\end{array}$ \\
\hline 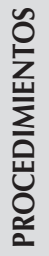 & $\begin{array}{l}\text { Desarrollar competencias } \\
\text { metodológicas (saber hacer)* } \\
\text { Desarrollar competencias de } \\
\text { dirección y trabajo en grupo } \\
\text { (liderazgo, toma de decisiones, etc,) } \\
\text { Adquirir destrezas relacionales } \\
\text { Adquirir destrezas comunicativas }\end{array}$ & $\begin{array}{l}\text { Adquirir habilidades para la elaboración de } \\
\text { trabajos en grupo } \\
\text { Adquirir habilidades para preparar los } \\
\text { exámenes } \\
\text { Adquirir destrezas relacionales }\end{array}$ \\
\hline
\end{tabular}

Cuadro 1. Objetivos específicos del proyecto.

\section{Estructura y elementos personales}

La Red de Mentores está basada en una estructura de tipo piramidal en cuyo vértice se sitúa el Coordinador General del Proyecto y cuya base está compuesta por los alumnos mentorizados (Fig. 1).

Los elementos o figuras que integran la estructura son de dos tipos: los que brindan su formación, experiencia y trabajo en beneficio de otros; y los receptores de la ayuda, destinatarios últimos de la acción. En el primer grupo están los que facilitan o ejercen la relación de ayuda, en términos de coordinación, formación y/o asesoramiento. En primer lugar, los coordinadores del proyecto, uno general (CG), que aparece el segundo año, cuando el proyecto se extiende a Madrid, y otros dos en cada una de las sedes, es decir, el coordinador del proyecto en la Universidad de Sevilla (CS) y el coordinador del proyecto en la Universidad Complutense de Madrid (CM). Estas tres figuras han sido claves para dar cohesión al proyecto; especialmente la del coordinador general cuya misión principal consistió en controlar que el proyecto se desarrollase de forma paralela en ambas sedes y de acuerdo al proyecto inicial. El coordinador general cobró un papel esencial de tutela directa en el desarrollo del proyecto en la Universidad Complutense dado que, como ya se ha indicado, se inició un año más tarde que en la Universidad de Sevilla.

* Se especifican en el apartado relativo a la formación teórica 
También forman parte del primer grupo los profesores tutores $(\mathrm{T})$ que, en el caso de Madrid, han sido cuatro profesores de la Facultad de Educación pertenecientes al Departamento de Métodos de Investigación y Diagnóstico en Educación, que ejercieron una función de asesoramiento y formación de los alumnos mentores. En conjunto tienen suficiente experiencia en el campo de la orientación (personal, familiar, escolar y profesional) y en el de la atención a la diversidad. Su misión consistió en desarrollar las sesiones de formación con los mentores y, tras ese período, tutorizarlos durante todo el proceso de mentorización. Para ello, se distribuyó a los mentores en cuatro grupos, asignando por tipo de titulaciones un grupo a cada profesor tutor. Estos se hacían cargo de un reducido grupo de mentores permitiéndoles atender, de una forma más personalizada y eficaz, las demandas que cualquiera de los alumnos que lo componían pudiese plantear a lo largo del proceso. A pesar de esta asignación inicial de los mentores a profesores tutores, quedó siempre abierta la posibilidad de comunicación de todos los mentores con cualquiera de los profesores tutores. Para hacerlo efectivo, la vía de comunicación fue triple: de forma presencial en horas de tutorías, por teléfono, o a través de una plataforma virtual, elemento esencial en todo el proceso.

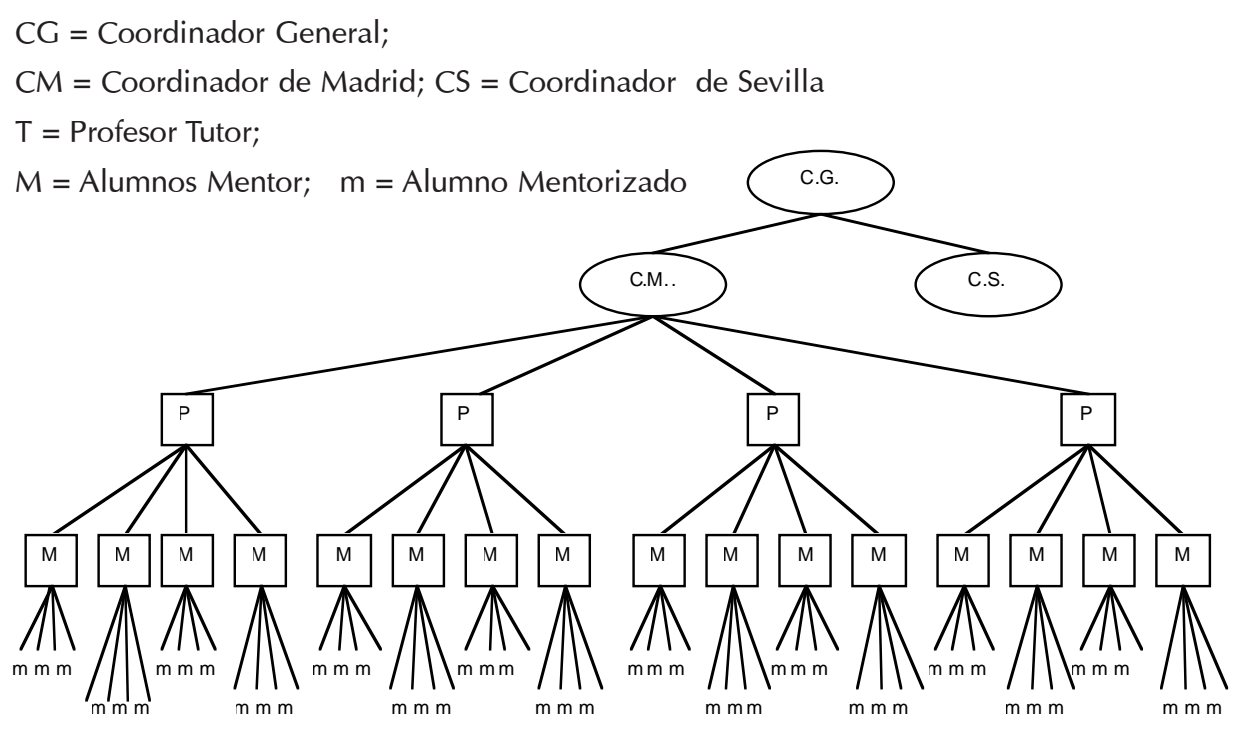

Figura 1. Estructura y elementos personales de la Red de Mentores

En el segundo grupo se encuentran los beneficiarios o receptores de la relación de ayuda, es decir, los alumnos mentorizados (m). Son alumnos de primer curso a los que se les ayuda en su incorporación a la vida universitaria. Los estudiantes mentorizados fueron reclutados por los mentores en la fase de captación. La adhesión de mentorizados al programa fue desigual en función de las titulaciones, consiguiendo un total de 125 estudiantes. 
Entre uno y otro grupo, a mitad de camino entre ambos, los que dan ayuda y los que la reciben, están los alumnos mentores (M), alumnos de últimos cursos matriculados en la asignatura genérica Red de Estudiantes mentores en la Universidad. Fueron quienes ejercieron, tras un período de formación y desde su función de mentores, la relación de ayuda directamente con los mentorizados.

Las titulaciones de procedencia de los estudiantes mentores fueron las siguientes: Maestro en Educación Musical, Maestro en Audición y Lenguaje, Pedagogía, Educación Social, Filología Inglesa, CC. Químicas, CC. Físicas, y Fisioterapia. Aunque, en un principio se contó con la participación de 22 mentores, hubo 8 que abandonaron por diversos motivos: 5 al principio del proceso (por no cumplir los requisitos, por tener ya suficientes créditos o por otras razones personales); y 3 a lo largo del proceso también por distintos motivos (sobrecarga de trabajo, bajo dominio en el uso del ordenador como herramienta, etc.) (Gráfico 1.).

$63 \%$

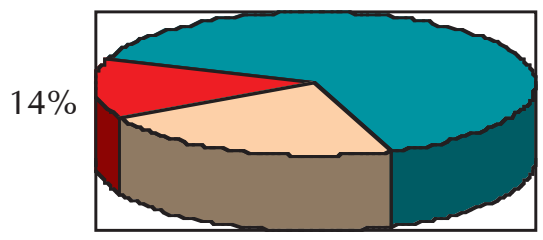

$23 \%$

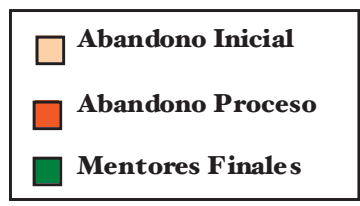

Mentores Finales

Gráfico 1. Distribución de los alumnos mentores inicialmente matriculados (\%)

Finalmente, se contó con la participación de 14 estudiantes mentores. En la tabla 1 se muestra un resumen de los participantes (mentores y mentorizados) por titulación.

\begin{tabular}{|c|c|c|}
\hline TITULACIÓN & $\begin{array}{c}N^{\circ} \text { DE } \\
\text { MENTORES }\end{array}$ & $\begin{array}{c}N^{\circ} \text { DE } \\
\text { MENTORIZADOS }\end{array}$ \\
\hline Filología Inglesa & 3 & 17 \\
\hline Ciencias Químicas & 2 & 7 \\
\hline Ciencias Físicas & 2 & 2 \\
\hline Pedagogía & 2 & 27 \\
\hline Magisterio & 3 & 53 \\
\hline Fisioterapia & 1 & 4 \\
\hline Educación Social & 1 & 15 \\
\hline Total & 14 & 125 \\
\hline
\end{tabular}

Tabla 1. Alumnos, mentores y mentorizados, participantes en el Proyecto (por titulación) 


\section{Modelo de intervención}

El modelo de intervención utilizado en REMUC es de tipo indirecto, concretamente el tradicionalmente denominado Modelo de Consulta (Bisquerra, 1998; Rodríguez Espinar, 1993). La principal característica de este modelo es que la relación de ayuda entre el profesional y el beneficiario de la misma es indirecta, lo que es posible gracias a una tercera figura, normalmente un para-profesional, que ejerce de mediador entre los dos anteriores elementos (Figura 2).

\section{Profesional de la relación de ayuda (PROFESOR TUTOR)}

\author{
Mediador en la \\ relación de ayuda \\ (MENTOR)
}
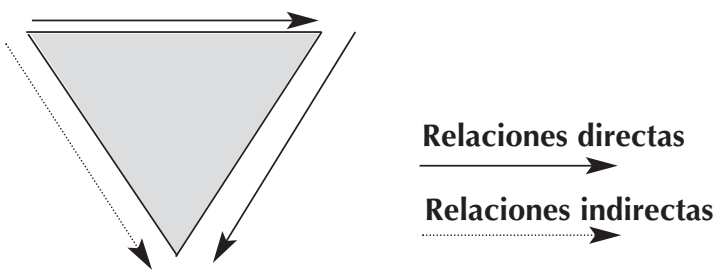

Beneficiario de la relación de ayuda (MENTORIZADO)

Figura 2. Modelo de Intervención de Consulta

En REMUC el profesional de ayuda es el profesor tutor, que es quien asesora y forma a los mentores. El mediador es el mentor, objeto del asesoramiento y formación por parte del profesor tutor y que será quien después asesore e informe a los mentorizados. Por último, el beneficiario de la relación de ayuda es el alumno mentorizado, que recibe directamente la ayuda del mentor.

\section{Soporte informático: el campus virtual de la mentoría}

Otro aspecto innovador en este programa consiste en el uso de una herramienta informática que otorgó al proceso una gran flexibilidad y, sobre todo, que permitió un trabajo colaborativo y continuo entre todos los agentes que intervinieron en el mismo, a pesar de la distancia física entre los participantes del programa.

La herramienta informática diseñada e implementada para el desarrollo del Proyecto tenía que ser lo suficientemente potente como para poder abordar toda la dimensión del mismo en cuanto a número de participantes, duración de la actividad, complejidad de las tareas de seguimiento y evaluación, etc. Esta consistió en un Portal de servicios, de uso exclusivo para los participantes profesionales y mediadores en el Proyecto (coordinadores, tutores y mentores) a través de una clave exclusiva de acceso, personalizando el acceso al perfil de cada uno de ellos (mentores o tutores). La gestión del diseño del portal la hizo el primer año del proyecto (200102) el equipo de la $U$. de Sevilla, si bien se introdujeron algunas mejoras en el curso 2002-03 en función de los resultados alcanzados el primer año. 
El portal incluye las siguientes utilidades (cuadro 2):

\begin{tabular}{|c|c|c|}
\hline $\begin{array}{l}\text { OPCIONES DE } \\
\text { COMUNICACIÓN }\end{array}$ & $\begin{array}{l}\text { OPCIONES DE } \\
\text { ESTUDIO }\end{array}$ & $\begin{array}{l}\text { OPCIONES DE } \\
\text { USUARIO }\end{array}$ \\
\hline $\begin{array}{l}\text { Sistema de mensajería inmediata } \\
\text { (SMI) } \\
\text { Correo personal } \\
\text { Foros de debate } \\
\text { Charlas on-line } \\
\text { Agenda-Calendario } \\
\text { Noticias }\end{array}$ & $\begin{array}{l}\text { Estudiar. Contenidos } \\
\text { formativos. } \\
\text { Autoevaluaciones. } \\
\text { Biblioteca } \\
\text { Grupos de Trabajo } \\
\text { Calificaciones }\end{array}$ & $\begin{array}{l}\text { Ficha personal } \\
\text { Compañeros } \\
\text { Grupos } \\
\text { Docentes }\end{array}$ \\
\hline
\end{tabular}

Cuadro 2. Utilidades incluidas en el portal web

\section{FASES DEL PROYECTO}

El desarrollo del proyecto en Madrid supuso un largo proceso que se concretó en las siguientes fases: toma de contacto con el proyecto desarrollado en Sevilla; gestión administrativa del proyecto para su realización en Madrid; campaña de difusión del proyecto; preparación, desarrollo e implementación del proceso y, por último, su evaluación.

\section{Fase $1^{\text {a }}$ : Toma de contacto y conocimiento del proyecto desarrollado en la Universidad de Sevilla.}

Debido a que la Universidad Complutense se incorpora a una experiencia que ya se había implementado en la Universidad de Sevilla el curso anterior (Valverde, 2001-02), lo primero que tuvo que hacer el equipo de Madrid fue conocer en profundidad dicha experiencia (objetivos, proceso, desarrollo, herramientas, materiales, resultados, etc.) para poder adaptarla e implantarla en su propia universidad (Valverde y otros, 2001 y 2002). En esta fase ejerció un papel fundamental el coordinador general.

\section{Fase $2^{\text {a }}$ : Gestión administrativa.}

Desarrollar el proyecto en Madrid implicó, como requisito inicial indispensable, gestionar la creación de una nueva asignatura genérica. Para ello fue necesario entrevistarse, en primer lugar, con el Decano de la Facultad, presentarle el proyecto y pedir, en la medida de lo posible, su apoyo. La asignatura genérica fue aceptada y se concedió un espacio físico para los encuentros dentro de la Facultad.

En segundo lugar, y dado que era un proyecto ínterfacultativo, solicitamos audiencia con el Vicerrector de Estudios para exponerle el proyecto. Su valoración fue altamente positiva y colaboró activamente en la aceptación de la asignatura, comprometiéndose a facilitar las condiciones pertinentes.

También se elaboró una carta dirigida a los Decanos de las Facultades o Escuelas en las que se iba a desarrollar la experiencia (Facultades y Escuelas a las que pertenecían los alumnos mentores y mentorizados), carta que se pensaba llevar en mano. 
Debido a lo precipitado del comienzo del proyecto, al final no fue posible hacerles entrega de la misma. ${ }^{1}$

Por último, una vez asegurada la posibilidad de realización, el proyecto fue presentado al Consejo del Departamento al que pertenecen los profesores tutores.

\section{Fase $3^{a}$ : Campaña de Difusión del proyecto.}

Al ser imprescindible para el desarrollo del proyecto el que hubiera alumnos que se matriculasen en la asignatura y al ser esta de nueva creación, fue necesario hacer una campaña de publicidad por el campus universitario. La premura con que se inicio el proyecto hizo que la difusión no fuese la deseada. No obstante, los 9 créditos asignados a la asignatura fue suficiente reclamo para obtener alumnos dispuestos a cursarla. Por otra parte, en este primer año no se deseaba un grupo excesivamente numeroso fijándose el cupo máximo de alumnos matriculados en la asignatura en 30.

\section{Fase 4a: Preparación del Proyecto.}

La fase de preparación por parte de los profesores tutores fue muy intensa. Aún cuando se contaba con la ventaja de un proyecto ya diseñado y experimentado, fue necesario realizar diversas reuniones con el fin de estudiar con detenimiento todo el proceso del proyecto, asignar funciones y tareas a los miembros del equipo, adaptar los documentos de los alumnos, establecer la temporalización, familiarizarse con la herramienta informática, etc.

En esta fase también se hizo un seguimiento del proceso de matriculación en la asignatura y se tuvo contacto individualizado con aquellos alumnos que, antes de matricularse en la asignatura, buscaron asesoramiento en relación con la misma.

\section{Fase $5^{\text {a }}$ : Implementación del proceso de mentoría y evaluación del mismo.}

La implementación del proceso, incluida la etapa de evaluación del mismo, se presenta desarrollada en toda su extensión en el siguiente apartado. Aclarar, no obstante, que el proceso de evaluación se ha realizado en dos sentidos: la evaluación del proceso de mentoría en tanto que proyecto y la evaluación del desempeño de los mentores con el fin de otorgarles una calificación en la asignatura.

El primer aspecto se ha evaluado utilizando diversas fuentes: índices de asistencia y de utilización de las herramientas de la plataforma virtual, número de los alumnos mentorizados y de sesiones realizadas; valoración individual de los mentores tanto de cada una de las sesiones de formación que se desarrollaron (se aplicaba un cuestionario que contemplaba aspectos relativos a utilidad y valoración), cuanto de una valoración del conjunto de la experiencia recogida en la memoria final que debían presentar. Así mismo, se ha podido obtener, de modo más informal, información día a día a partir de las opiniones expresadas en las sesiones presenciales o a través de los comentarios volcados en cualquiera de las herramientas de comunicación del campus virtual.

1. Este es uno de los aspectos que hay que corregir en la implantación en el curso 2003-04. 
El segundo aspecto, evaluación de la actuación de los mentores en el proyecto, se ha realizado a través de la confección de la memoria final elaborada de forma individual por los alumnos mentores así como por sus índices de participación en las sesiones presenciales y en los encuentros virtuales a través del portal web. En ocasiones también mediante las tutorías demandadas por el propio alumnado.

\section{DESARROLLO DEL PROYECTO}

El proceso se concretó en cuatro etapas: una primera de formación teórica inicial de los alumnos mentores; una segunda de captación de los alumnos mentorizados, y la tercera y cuarta consistieron en el desarrollo y evaluación del proceso de mentorización, respectivamente. La última etapa -evaluación del REMUC y resultados- se describe en apartado independiente.

\section{Formación teórica inicial del mentor}

La formación teórica de los mentores se realizó a lo largo de 4 sesiones presenciales, organizadas durante los meses de octubre a diciembre de 2002, impartidas por los profesores tutores, y con una periodicidad semanal. Tras una sesión inicial introductoria con el propósito de crear conciencia de grupo e introducirles en el objetivo del proyecto, se desarrollaron otras tres sesiones en las que se contemplaron con más detalle contenidos teóricos sobre el proceso de mentoría y otros más prácticos relativos al desarrollo de su función de mentores: estrategias para la captación de mentorizados, el proceso de mentorización de estudiantes de nuevo ingreso, y entrenamiento en el uso del campus virtual. (Cuadro 3).

\begin{tabular}{|l|c|l|l|}
\hline Fecha & Asistenc. & Contenido & Actividad / Material \\
\hline $13 / 11 / 02$ & 16 & $\begin{array}{l}\text { Mentoría } \\
\text { Recogida de datos } \\
\text { Presentación de los } \\
\text { participantes }\end{array}$ & $\begin{array}{l}\text { Presentación Power Point } \\
\text { Fotopalabra } \\
\text { Cuestionario } \\
\text { Hoja de evaluación }\end{array}$ \\
\hline $20 / 11 / 02$ & 16 & $\begin{array}{l}\text { Rol Mentor } \\
\text { Conciencia de equipo }\end{array}$ & $\begin{array}{l}\text { Casos } \\
\text { Ciego (dinámica) } \\
\text { Hoja de evaluación }\end{array}$ \\
\hline $27 / 11 / 02$ & 14 & $\begin{array}{l}\text { Conocimiento del SIMUC } \\
\text { Aula de informática } \\
\text { Práctica }\end{array}$ \\
\hline $4 / 12 / 02$ & 14 & $\begin{array}{l}\text { Relación de ayuda } \\
\text { Proceso de captación }\end{array}$ & $\begin{array}{l}\text { Lluvia de ideas } \\
\text { Documento 4 } \\
\text { Guía básica de captación }\end{array}$ \\
\hline
\end{tabular}

Cuadro 3. Síntesis de las sesiones formativas

Los objetivos de formación de los estudiantes mentores estuvieron relacionados con el desarrollo de una serie de competencias que se detallan en el cuadro 4. 
En el Proyecto se establecieron relaciones verticales y horizontales, ambas alrededor del concepto de equipo. Las relaciones verticales se realizaron de la siguiente manera: cada tutor trabajó con sus alumnos mentores (constitución de los equipos tutor-mentores), con los que se reunió aproximadamente una vez al mes atendiendo sus dudas y preguntas. ${ }^{2}$ Cada mentor, a su vez, tenía a su cargo un grupo de alumnos mentorizados (constitución de los equipos mentor-mentorizados), con los que se reunía aproximadamente una vez a la semana.

De forma complementaria a esta estructura vertical, se definieron también relaciones horizontales para que la información pudiese fluir entre iguales. Así, se crearon equipos virtuales, de tutores y mentores entre si (equipo de tutores y equipo de mentores, respectivamente) utilizando como vía de comunicación preferente las herramientas creadas a tal fin en el portal web (foros de debate, chats y correo electrónico) si bien, en el caso de los mentores, también celebraron encuentros por grupos más o menos homogéneos en sus propios centros y en el de los tutores, por estar en la misma facultad, encuentros periódicos. De hecho, una de las recomendaciones era que los mentores de la misma Facultad trabajaran en equipo. De hecho así sucedió en la Facultades de Educación y Filología.

\begin{tabular}{|c|c|c|}
\hline $\begin{array}{l}\text { COMPETENCIA TÉCNICA } \\
\text { (SABER) }\end{array}$ & $\begin{array}{l}\text { COMPETENCIA } \\
\text { METODOLÓGICA } \\
\text { (SABER HACER) }\end{array}$ & $\begin{array}{c}\text { COMPETENCIA SOCIAL } \\
\text { Y PARTICIPATIVA } \\
\text { (SABER SER Y ESTAR) }\end{array}$ \\
\hline $\begin{array}{l}\text { Conocimiento del perfil del } \\
\text { alumnado de nuevo ingreso }\end{array}$ & $\begin{array}{l}\text { Gestión y dinamización de } \\
\text { grupos. }\end{array}$ & $\begin{array}{l}\text { Respeto a principios éticos de } \\
\text { la actuación del mentor. }\end{array}$ \\
\hline $\begin{array}{l}\text { Conocimiento de la problemática } \\
\text { de la Orientación en la Universidad. } \\
\text { Tutorías. Sistema de Apoyo al } \\
\text { Estudiante. Programas de Orientación } \\
\text { Comprensión del proceso de } \\
\text { mentoría de alumnos en la Univer- } \\
\text { sidad. Sistemas de Mentorias. La } \\
\text { mentoría por compañeros. }\end{array}$ & $\begin{array}{l}\text { Entrevista de Orientación. } \\
\text { Identificación de necesidades } \\
\text { de los alumnos de primero. } \\
\text { Dominio de las técnicas de } \\
\text { comunicación. } \\
\text { Establecimiento de la relación } \\
\text { mentor-mentorizado. }\end{array}$ & $\begin{array}{l}\text { Compromiso con el cambio y } \\
\text { la mejora de la calidad de } \\
\text { enseñanza. } \\
\text { Colaboración. } \\
\text { Escucha activa. }\end{array}$ \\
\hline $\begin{array}{l}\text { Comprensión de las fases del ciclo } \\
\text { de la mentoría: captación, análisis } \\
\text { de necesidades, planificación de la } \\
\text { actuación, evaluación. } \\
\text { Conocimiento de Proyecto Mentor: } \\
\text { Objetivos. Contenidos. Desarrollo. } \\
\text { Evaluación. }\end{array}$ & $\begin{array}{l}\text { Procedimientos de resolución } \\
\text { de problemas. } \\
\text { Organización y planificación } \\
\text { del trabajo. } \\
\text { Utilización de herramientas } \\
\text { y recursos específicos para la } \\
\text { orientación del aprendizaje y } \\
\text { la orientación profesional. } \\
\text { Búsqueda y gestión de la } \\
\text { información. } \\
\text { Procedimientos de evaluación. }\end{array}$ & \\
\hline
\end{tabular}

Cuadro 4. Competencias a desarrollar en los mentores

2. En ningún momento se descartó la conexión de cualquier mentor con cualquier tutor o viceversa, relación establecida fundamentalmente a través de la plataforma virtual. 
A lo largo del desarrollo de la parte práctica de la asignatura (enero-junio) y con el fin de ir evaluando el proceso y solucionar dudas y problemas que pudieran surgir, se celebraron dos sesiones presenciales más, en este caso de seguimiento (cuadro 5). ${ }^{3}$ También fue necesario tratar, en la última sesión presencial, por demanda de los propios mentores, aspectos relativos a la evaluación de la asignatura. En ella se explicaron los elementos que se tendrían en cuenta y el peso específico de cada uno. También se les orientó en la elaboración de la memoria detallando los contenidos mínimos que debería tener y la estructura que debía seguir. Esta actividad fue especialmente requerida por los alumnos no pertenecientes a la Facultad de Educación, ya que manifestaron abiertamente no saber cómo realizar una memoria.

\begin{tabular}{|l|c|l|l|}
\hline FECHA & ASISTENC. & \multicolumn{1}{|c|}{ CONTENIDO } & ACTIVIDAD / MATERIAL \\
\hline $26 / 02 / 03$ & 2 & $\begin{array}{l}\text { Seguimiento } \\
\text { Solución de problemas }\end{array}$ & $\begin{array}{l}\text { Mantel giratorio } \\
\text { (dinámica) }\end{array}$ \\
\hline $12 / 03 / 03$ & 13 & $\begin{array}{l}\text { Seguimiento } \\
\text { Solución de problemas }\end{array}$ & $\begin{array}{l}\text { Descripción proceso } \\
\text { individual de mentoría } \\
\text { Memoria }\end{array}$ \\
\hline
\end{tabular}

Cuadro 5. Sesiones de seguimiento

\section{Proceso de Captación de los alumnos mentorizados}

Este fue uno de los aspectos críticos de cara a garantizar una buena puesta en marcha del Proyecto. Se hizo necesaria una fase de divulgación y publicidad del mismo. Este proceso, al que se llamó captación, fue llevado a cabo por los propios estudiantes mentores a través de:

- Carteles Informativos colocados en las clases de primero en los que se informaba sobre la experiencia a desarrollar y sobre las ventajas que podría tener si participara; y

- Trípticos con información acerca de lo que significa la mentoría en la Universidad, qué es el Proyecto Mentor, cuáles son sus objetivos, qué es un alumno mentorizado y qué beneficios puede obtener con su participación.

Estas herramientas fueron utilizadas por el grupo de estudiantes mentores pertenecientes al mismo centro de forma coordinada. Con el fin de lograr su objetivo, captar alumnos de primero, algunos mentores contactaron con profesores de primero para solicitarles permiso para exponer en una de sus clases el Proyecto (actividad que como termino medio no ocupó más de 10 ó 15 minutos). Cuando así se hizo, el profesorado resultó ser un elemento especialmente motivador para que el alumnado se

3. Una de ellas hubo que repetirla debido a que, por un problema en la convocatoria, el numero de asistentes fue muy bajo². 
decidiera a inscribirse como mentorizado. En ocasiones también se recurrió a otras estrategias muy ligadas al tipo de facultad: acudir a las academias a las que asisten los alumnos, reunirse con los equipos deportivos, etc.

En todos los casos, tras una primera sesión en la que se les explicaba el proyecto, se firmaban los contratos (documento por el que el mentorizado se comprometía a seguir en el proyecto hasta el final de curso). El enganche inicial de los alumnos fue muy dispar variando en función de las titulaciones. En algunos casos, una vez iniciado el proceso, se apuntaron nuevos mentorizados atraídos por lo que sus compañeros comentaban.

\section{Desarrollo del proceso de mentorización}

Una vez realizada la captación de estudiantes, los estudiantes mentores se dedicaron a la detección de necesidades de los alumnos mentorizados. Contaban para ello con un completo temario de trabajo organizado teniendo en cuenta, fundamentalmente, la importancia relativa de los temas y su conveniencia en función del calendario. Se garantizaba así que todos los alumnos tutelados recibiesen la misma información sobre los temas que se consideraban más importantes (aquellos temas que más pudieran necesitar o interesar a los alumnos de nuevo ingreso) y que los abordasen en el momento más adecuado.

La propuesta no obstante no era cerrada. Al margen de los temas propuestos era previsible que surgieran nuevos temas y preguntas a tratar en cada reunión y equipo. Se pretendía que la dinámica se ajustase lo más posible a las características de cada grupo de mentorizados. Cada mentor debía estructurar los temas y el tiempo a las necesidades e intereses suscitadas en su grupo, si bien se le pedía que no se apartase en exceso de las directrices marcadas. Especialmente se les sugería que respetasen la temporalización en aquellos temas que eran especialmente pertinentes en un momento determinado del curso. El documento ofrecía unas pautas básicas de modo que se dejase libertad para que los mentores alterasen el orden, contenido y/o la profundidad de los temas tratados.

El principal procedimiento utilizado en la detección de necesidades fueron las técnicas de dinámica de grupo.

En cada equipo mentor-mentorizados las necesidades fueron surgiendo de modo desigual. En general, lo hicieron a medida que se desarrollaban las sesiones de intervención. En ocasiones, al principio parecía no haberlas; en estos casos fueron los mentores los que las suscitaron a través de preguntas. En general, cuando se fueron acercando los exámenes, se hicieron explícitas bastantes necesidades relativas a cómo afrontarlos. De hecho, algunos grupos que empezaron con sus alumnos un poco más tarde debido a que la fase de captación les resultó más difícil, aprovecharon ese momento crítico, el de los exámenes, para hacer sentir a sus compañeros la utilidad de la mentoría. ${ }^{4}$

Las necesidades detectadas por los mentores giraron en torno a los tres grandes ámbitos de la orientación: personal, académica y profesional, con predominio de estas dos últimas (cuadro 6):

4. Esto sucedió especialmente en las titulaciones de ciencias: CC Químicas y CC Físicas. 


\begin{tabular}{|c|c|c|}
\hline PERSONAL & ACADÉMICA & PROFESIONAL \\
\hline $\begin{array}{l}\text { Alta Presión y dedicación } \\
\text { exclusiva al estudio (sobre todo } \\
\text { en las carreras técnicas), que } \\
\text { dificulta el desarrollo de otras } \\
\text { facetas personales, culturales, } \\
\text { sociales, de participación en la } \\
\text { vida universitaria. }\end{array}$ & $\begin{array}{l}\text { Desconocimiento de los Planes } \\
\text { de Estudio en general: itinerarios } \\
\text { formativos, optatividad, tipos de } \\
\text { asignaturas... } \\
\text { Necesidades relativas al estudio: } \\
\text { planificación del estudio, necesitan } \\
\text { de mucho tiempo y de dedicación, } \\
\text { problemas para adaptarse a la } \\
\text { metodología docente (las anteriores } \\
\text { necesidades dentro de este punto, } \\
\text { se dan sobre todo en las carreras } \\
\text { técnicas), preparación de exámenes. } \\
\text { En la realización de tareas buro- } \\
\text { cráticas-administrativas: ya que no } \\
\text { conocen los planes de estudio, ni } \\
\text { los tipos de asignaturas (libre } \\
\text { configuración, optativas, } \\
\text { cuatrimestre...). } \\
\text { Desconocimiento de los Servicios } \\
\text { y Recursos, Becas y Ayudas que } \\
\text { posee el centro en particular y la } \\
\text { Universidad en General. } \\
\text { Escasa valoración de la función } \\
\text { tutorial del profesorado. }\end{array}$ & $\begin{array}{l}\text { Poca planificación y toma de } \\
\text { decisiones: escasa preparación } \\
\text { para configurar sus propios } \\
\text { itinerarios formativos, para } \\
\text { tomar decisiones en relación a } \\
\text { optativas y libre configuración. } \\
\\
\text { Necesidades relativas a las } \\
\text { salidas profesionales: falta de } \\
\text { información, carencia de visión } \\
\text { respecto a la proyección } \\
\text { profesional de la titulación. } \\
\text { Profesionalización de la } \\
\text { enseñanza universitaria: } \\
\text { exceso de contenidos poco } \\
\text { aplicados, pocas prácticas, } \\
\text { escasa relación con el } \\
\text { ejercicio profesional. }\end{array}$ \\
\hline
\end{tabular}

Cuadro 6. Necesidades detectadas en los mentorizados

El trabajo de los mentores se planificó de forma que siguieran las etapas propias de todo proceso de mentoría intencional y formal (figura 3) (Valverde Macias, García Jiménez, Ruiz de Miguel y Romero Rodríguez 2003):

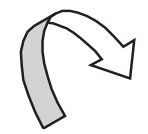

Experiencia
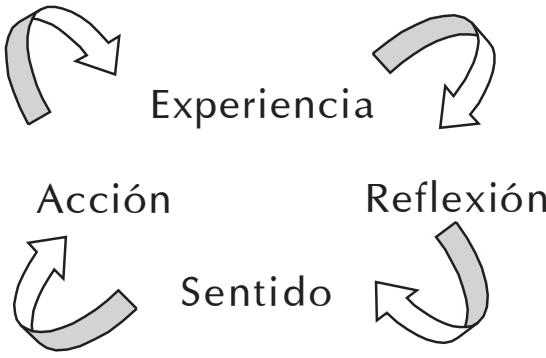

Figura 3. Proceso de mentoria

Tras las primeras sesiones, en las que se consolidó el grupo y se analizaron las necesidades, se desarrolló un proceso en el que el mentor planteaba una experiencia relacionada con la problemática a abordar que después era analizada en profundidad. Una vez finalizado este análisis se planteaba a los mentorizados el significado que para ellos había tenido la experiencia y cómo la podían traducir en una acción que diera respuesta al problema que les preocupaba. El papel del mentor era, por tanto, de facilitador de experiencias de aprendizaje y no de consejero. 
Se insistió mucho en la creación de un clima colaborativo y en una comunicación fluida y espontánea entre los alumnos mentores y mentorizados. Esto no fue posible en todos los grupos, debido a sus propias características o a la falta de experiencia de los mentores. En este sentido se apreciaron grandes diferencias entre los mentores provenientes de la Facultad de Educación y del resto de Facultades.

Las primeras sesiones de mentoría se dedicaron a la toma de contacto y a crear conciencia de grupo, si bien la estrategia de romper el hielo normalmente había que retomarla al comienzo de cada sesión. En los demás encuentros se fueron abordando las necesidades detectadas, siguiendo un programa previo o en función de las que iban surgiendo día a día. Las sesiones o encuentros entre los mentores y los mentorizados oscilaban de entre hora y media a dos horas.

\section{EVALUACIÓN DEL REMUC (2002-2003): RESULTADOS}

De la primera revisión de las evaluaciones realizadas se desprende un resultado realmente positivo en la apreciación del Proyecto en sí, tanto en cuanto a su desarrollo, como al papel de los participantes y los beneficios generales y personales recibidos.

\section{Formación inicial del mentor}

En cada sesión formativa se pidió la opinión al mentor a través de un cuestionario. El cuadro 7 resume la valoración dada por los mentores sobre las sesiones de formación. La escala utilizada fue de 1 a 5 obteniéndose una media en utilidad de 4,6 y en valoración general de 4,2. En términos cualitativos los puntos fuertes atribuidos a las sesiones formativas fueron: aspectos de aprendizaje (conocimientos y estrategias); el ambiente de clase (ameno); el modo de trabajar (trabajo en equipo, nivel de participación y carácter práctico de la sesión). En las primeras sesiones predominó fundamentalmente la valoración positiva de los aspectos de aprendizaje y en las últimas las del modo de trabajar.

\begin{tabular}{|c|c|l|l|l|c|c|}
\hline Sesión & Asist. & Contenido & Puntos fuertes & Puntos débiles & $\begin{array}{c}\text { Utilidad } \\
\text { (sobre 5) }\end{array}$ & $\begin{array}{c}\text { Valoración } \\
\text { (sobre 5) }\end{array}$ \\
\hline 1 & 16 & $\begin{array}{l}\text { Mentoría } \\
\text { Recogida de datos } \\
\text { Presentación de } \\
\text { los participantes }\end{array}$ & $\begin{array}{l}\text { Conocimientos } \\
\text { adquiridos } \\
\text { Ambiente de clase } \\
\text { Estrategias aprendidas }\end{array}$ & $\begin{array}{l}\text { Tiempo excesivo } \\
\text { Inseguridad }\end{array}$ & 4,1 & 4 \\
\hline 2 & 16 & $\begin{array}{l}\text { Rol Mentor } \\
\text { Conciencia de } \\
\text { equipo }\end{array}$ & $\begin{array}{l}\text { Trabajo en grupo } \\
\text { Participación } \\
\text { Ambiente }\end{array}$ & $\begin{array}{l}\text { Tiempo excesivo } \\
\text { Cambio de espacio }\end{array}$ & 4,6 & 4,3 \\
\hline 3 & 14 & $\begin{array}{l}\text { Conocimiento } \\
\text { del SIMUC }\end{array}$ & $\begin{array}{l}\text { Práctico } \\
\text { Ameno }\end{array}$ & $\begin{array}{l}\text { Problemas técnicos } \\
\text { en el portal }\end{array}$ & 4,2 & 4,3 \\
\hline $4^{2}$ & 14 & $\begin{array}{l}\text { Relación de ayuda } \\
\text { Proceso de } \\
\text { captación }\end{array}$ & Sin datos & Sin datos ${ }^{2}$ & Sin datos & Sin datos ${ }^{2}$ \\
\hline
\end{tabular}

Cuadro 7. Resultados de la evaluación de las sesiones de formación

NOTA: 1 Hubo problemas con el aula y fue necesario cambiar durante la sesión

2 En esa sesión no se aplicó cuestionario de evaluación 


\section{Proceso de captación y mentorización}

El desarrollo de las sesiones de mentorización fue muy dispar (tabla 2). El número de mentorizados captados oscilo de 1 a 42 (grupo clase); el de sesiones realizadas de 4 a 11. También varió la asistencia de los alumnos mentorizados ya que no todos asistieron con asiduidad lo que hizo que bajase considerablemente el número de asistentes permanentes. Destacar que en las titulaciones de CC. Físicas y Químicas es dónde hubo el menor número de mentorizados y donde más hubo fue en Educación Social y Pedagogía. Destaca el caso de Audición y Lenguaje que fue, con diferencia, la titulación que más alumnos mentorizados tuvo y más sesiones realizó.

\begin{tabular}{|l|c|c|}
\hline PROCEDENCIA & $\begin{array}{c}\text { ESTUDIANTES } \\
\text { MENTORIZADOS }\end{array}$ & SESIONES REALIZADAS \\
\hline Filología Inglesa 1 & 6 & 4 \\
\hline Filología Inglesa 2 & 6 & Sin datos \\
\hline Filología Inglesa 3 & 5 & 7 \\
\hline Educación Musical 1 & 5 & 5 \\
\hline Educación Musical 2 & 6 & 11 \\
\hline Audición y Lenguaje & 42 & $4^{3}$ \\
\hline Pedagogía 1 & $12^{3}$ & $4^{3}$ \\
\hline Pedagogía 2 & $12^{3}$ & $7^{3}$ \\
\hline Educación Social & $15^{3}$ & 8 \\
\hline Fisioterapia & 4 & $4^{2}$ \\
\hline CC. Químicas 1 & $6^{2}$ & $4^{2}$ \\
\hline CC. Químicas 2 & $1^{2}$ & $6^{1}$ \\
\hline CC. Físicas 1 & $2^{1}$ & $6^{1}$ \\
\hline CC. Físicas 2 & $2^{1}$ & \\
\hline
\end{tabular}

Tabla 2. Número de estudiantes mentorizados y sesiones realizadas por cada mentor según procedencia

Los mentores destacaron el doble apoyo (presencial y por internet) que tuvieron por parte de los tutores y a través de las herramientas facilitadas por ellos.

Un problema que acusaron fue el de los horarios para coincidir entre tutores y mentores (menos acusado), entre mentores (presencial o en el chat) y entre mentores y mentorizados (el más acusado). En este sentido muchos adoptaron el recurso de internet para comunicarse con sus mentorizados, como complemento al contacto presencial. La repercusión que esta incompatibilidad de horarios produjo se hizo sentir en la falta de constancia en la asistencia a las sesiones lo que dificultó la mar-

NOTAS: 1 Compartidos; 2 Compartidos; 3 Mentores trabajando en equipo pero de forma separada con el grupo de Pedagogía (12 alumnos y 4 sesiones) y con el de Educación Social (15 alumnos y 7 sesiones). 
cha de las mismas (no siempre venían los mismos por lo que el grupo estaba continuamente cambiando).

Por último, señalar que el hecho de que la experiencia se llevase por primera vez en la UCM se ha hecho notar en la acomodación a los medios del portal y al funcionamiento del proceso en los tutores, redundando en los mentores y retrasando un poco la marcha del proceso.

\section{Utilización del Campus Virtual}

La utilización de las herramientas del portal fue muy desigual (tablas 3a y 3b). A continuación se explican las herramientas más utilizadas.

\begin{tabular}{|l|c|c|c|c|c|c|c|c|c|c|}
\hline \multicolumn{10}{|c|}{ HERRAMIENTAS DEL PORTAL } \\
\hline & $\begin{array}{c}\text { Inicios de } \\
\text { sesión }\end{array}$ & $\begin{array}{l}\text { Páginas } \\
\text { visitadas }\end{array}$ & Portada & SMI & Correo & Foro & Charlas & Agenda & Noticias & Estudiar \\
\hline TOTAL & 1704 & 9510 & 3577 & 1086 & 478 & 3026 & 169 & 188 & 46 & 150 \\
MEDIA & 126 & 679,28 & 255,5 & 77,78 & 36,76 & 216,14 & 13 & 13,42 & 3,28 & 10,71 \\
MAX & 449 & 1706 & 640 & 354 & 71 & 623 & 47 & 31 & 10 & 25 \\
MIN & 9 & 47 & 11 & 2 & 0 & 1 & 0 & 2 & 0 & 1 \\
\hline
\end{tabular}

Tabla 3a. Utilización de las herramientas del portal

\begin{tabular}{|l|c|c|c|c|c|c|c|c|c|c|c|}
\hline \multicolumn{10}{|c|}{ HERRAMIENTAS DEL PORTAL (continuación) } \\
\hline & Autoeva & Biblioteca & $\begin{array}{c}\text { Grupos } \\
\text { Trabajo }\end{array}$ & Califica & Ficha & Compis & Grupos & Docentes & Ayuda & $\begin{array}{c}\text { Esta- } \\
\text { disticas }\end{array}$ & $\begin{array}{c}\text { Presen- } \\
\text { ciales }\end{array}$ \\
\hline TOTAL & 59 & 90 & 48 & 33 & 134 & 167 & 35 & 30 & 23 & 98 & 70 \\
\hline MEDIA & 4,21 & 6,92 & 3,42 & 2,36 & 9,57 & 12,85 & 2,92 & 2,31 & 3,28 & 7 & 5,38 \\
\hline MAX & 16 & 16 & 12 & 6 & 34 & 38 & 7 & 5 & 9 & 17 & 13 \\
\hline MIN & 1 & 0 & 1 & 1 & 0 & 0 & 0 & 0 & 0 & 1 & 0 \\
\hline
\end{tabular}

Tabla 3b. Utilización de las herramientas del portal

A) Foros de discusión: se desarrollaron distintos foros a lo largo del curso, en función de la etapa del proceso en la que se estaba y de las necesidades que fuesen surgiendo en los mentores: Caminando por el campus, Captación de alumnos, Desarrollo de las sesiones, etc.

b) Estudiar: apartado donde se encontraban recogidos todos los materiales para la formación y el proceso de mentorización. Puede considerarse un banco de recursos y actividades abierto a las necesidades de los mentores, y que se iba completando y mejorando a lo largo del curso, con aquellos materiales que los tutores creían pertinentes o que algunos mentores consideraban importante que también lo tuvieran otros compañeros mentores. En este sentido la Universidad Complutense aportó algunos documentos relativos a partes del proceso y sobre dinámicas de grupo. 
c) Correo personal (interno del portal): la herramienta más potente para la comunicación con el alumnado en materia de dudas, sugerencias, entrevistas, materiales, etc.

d) Chats: se desarrollaron diversos chats semanales relacionados con la fase del proceso en la que se estaba y de acuerdo con las necesidades e interesesque surgían. En concreto, la dinámica en su desarrollo fue que el tutor planteaba diversos asuntos, y se iban abordando por los distintos mentores participantes. Uno de los grandes inconvenientes en su desarrollo, fue los problemas para concretar un horario de encuentro ya que, al ser mentores de diferentes centros y, consiguientemente, con distintos horarios de clase y de estudio, era difícil coincidir. A cada uno de los chats no solían asistir más de 7 alumnos/as.

E) Agenda y noticias: a través de estas dos herramientas (además de los foros) el alumno estuvo en todo momento informado de acontecimientos que se iban a realizar: sesiones presenciales, fechas importantes en relación con la Universidad, etc. Finalmente comentar que, aunque el alumnado también tenía la posibilidad de aportar noticias, no se registró prácticamente ninguna.

f) Compañeros: A través de esta opción, se facilitó la posibilidad de conocer de donde provenían los mentores y tener algunas notas de referencia de cada uno: hobbies, estudios, correo electrónico personal (externo a la red del campus, etc.)

\section{Logro de objetivos}

\section{- En los mentorizados}

No ha habido un control directo de los logros obtenidos por los alumnos mentorizados (cuestionario o entrevista). La única fuente utilizada ha sido los comentarios de los propios mentores. En ese sentido es escasa la información obtenida aunque muy contundente la valoración expresada por los mentores. Éstos reconocen que los alumnos mentorizados están ahora en mejores condiciones que cuando ellos estuvieron en primero, pues a ellos nadie los ayudó. Esta ayuda es, según ellos, muy necesaria.

Otra evidencia de la positiva valoración es la sugerencia que los mentores realizan en las memorias: proponen que la experiencia se extienda a todos los alumnos y a todos los cursos posibles o, al menos, que llegue también a los alumnos de segundo curso y/o a los que se matriculan en primero por segunda vez.

Entre los logros relativos a los mentorizados que los mentores señalan cabe destacar:

- creación entre los mentorizados de un compañerismo y una visión de colaboración hasta el momento inexistente

- ayuda al alumnado al inicio de la vida universitaria

\section{- En los mentores}

Al ser la única fuente de comprobación de logros los alumnos mentores, es en los logros relativos a ellos mismos de lo que más información se posee.

Los mentores han definido la asignatura como un programa experimental de apoyo a los alumnos de $1^{\mathrm{er}}$ curso de carreras universitarias por parte de los alumnos 
de últimos cursos de esas mismas carreras que se adhieren al programa a través de su matriculación en una asignatura de libre configuración y en este sentido la califican de muy formadora.

Entre los logros relacionados con los mentores se señalan los siguientes:

- creación entre los mentores de la misma o de distinta facultad de un compañerismo y una visión de colaboración hasta el momento inexistente;

- apoyo y entusiasmo mutuo entre mentores a pesar de ser un grupo que inicialmente no se conocía;

- intercambio de ideas entre alumnos de distintas facultades;

- ayuda en el desarrollo de habilidades sociales y de actitudes pedagógicas sobre todo en los alumnos de ciencias;

- acercamiento a las nuevas tecnologías y toma de conciencia del uso que se le puede dar a internet como herramienta de trabajo entre profesor y alumno;

- flexibilidad que el sistema otorga a la hora de trabajar y permitir estar en contacto de forma directa con tutores y mentorizados;

- creación de un grupo que se autogestiona;

- interactuar con futuros colegas de profesión y comprobar los cambios generacionales;

- descubrimiento de otra forma de trabajar en la Universidad;

- detección temprana de problemas y deficiencias en la actividad diaria del centro universitario;

- conocimiento de itinerarios formativos;

- aprender a compartir y relativizar los propios problemas;

- refuerzo de alumnos de la propia titulación para reivindicar mejoras en la docencia del centro;

- $\quad$ aprendizaje para gestionar grupos y planificar su trabajo.

Por último cabe destacar una serie de incidencias que ocurrieron a lo largo del desarrollo del proceso, que han estado marcadas por tres hechos: la dispersión de los mentores en diferentes centros de la universidad; la ausencia de espacios asignados para reunirse en las distintas facultades y de horarios comunes; el número de sesiones presenciales condicionadas por la posibilidad de interaccionar a través de la web; y un uso insuficiente (por debajo de lo esperado) de los recursos que la plataforma virtual ofrecía. Como consecuencia de estas premisas, habría que destacar:

- Problemas de horarios y espacios: debido al exceso de clases que tienen los alumnos, en especial los mentores, y también por no disponer de un espacio fijo para las sesiones de mentorización. Unido a ello, la falta de constancia en la asistencia de los estudiantes mentorizados.

- Para la mayoría de los mentores la formación presencial fue insuficiente; consideran que hubieran necesitado una formación más amplia para enfrentarse posteriormente al proceso de mentoría y, según algunos, más intensiva o concentrada. Esta necesidad se hizo mas patente en los alumnos de las Facultades de Ciencias. No obstante, para otros mentores, la formación recibida fue la adecuada y les otorgó suficiente autonomía. En cualquier caso, si bien los 
alumnos valoran como útiles las sesiones formativas, perciben que es en el desarrollo de la mentoría cuando realmente aprenden.

- Por otro lado, y dentro de lo que hace referencia a la herramienta informática, cabe destacar que los mentores acusan una falta de hábito del uso de internet de manera generalizada así como dificultad de acceso por no disponer de él en su casa. En este sentido los mentores reclaman que se les informe de los requisitos técnicos de la asignatura, es decir, poseer un dominio mínimo del uso internet y posibilidad de acceso. Señalan también algunas mejoras que podrían introducirse en el portal, en los foros y chat principalmente, por ser las herramientas más utilizadas.

- Necesidad de un mayor control y seguimiento por parte del profesorado.

Un problema de difícil solución sigue siendo el ajuste del calendario de la convocatoria de la asignatura genérica de libre elección, el desarrollo de los cursos de formación y el inicio de la actividad de mentoría, aunque, por otra parte, habría que destacar que el alumnado reconoce la necesidad de extender los procesos de mentoría a otros cursos y problemáticas universitarias.

\section{CONCLUSIÓN Y PROSPECTIVA}

Como conclusión, puede señalarse que, a pesar de las dificultades encontradas para llevar a cabo el Proyecto y el estado incipiente en el que se encuentra, éste parece aportar interesantes beneficios tanto para los estudiantes mentores como para los mentorizados. Además se presenta como un proceso que podría ayudar a extender la orientación en la universidad, haciéndose más cercana al alumnado. Este proceso, además, podría atender a necesidades muy diversas que se producen en el seno de nuestras universidades.

Por estas razones, puede considerarse que la mentoría se configura como un elemento de mejora de la calidad de la enseñanza universitaria y de desarrollo de competencias profesionales que serán requeridas a nuestros futuros titulados para poder competir en el entorno europeo. A modo de ejemplo y para concluir, transcribimos algunas palabras textuales de los propios mentores:

"la experiencia nos ha enriquecido a todos y nos ha hecho ver que la UCM va mas

allá de tu facultad y que el compañerismo va más allá de tu grupo de clase";

"me ha ayudado a ver que fuera de la facultad hay otro modo de ver las cosas";

"me ha ayudado a valorarme más como persona y como profesional";

"ha fortalecido nuestra autonomía y las destrezas de organización";

"nos ha ayudado a superar problemas que hasta entonces no te dabas cuenta que tenías"; "nos ha servido de aprendizaje también sobre nosotros mismos y nuestro carácter"; "al ayudar a los demás anticipando problemas te ayudas a ti mismo a replanteártelos ya que sus problemas futuros pueden ser tus problemas presentes";

por otro lado, "ha creado en nosotros un sentimiento de satisfacción por sentirnos útiles"; y, por último, "nos ha obligado a pensar mucho". 
La experiencia continúa en el curso 2003-2004, donde se ha vuelto a ofertar la asignatura genérica con las mismas características, tratando de solventar los problemas encontrados en la convocatoria del curso anterior: se ha profundizado en la formación inicial de los mentores, ofreciendo contenidos más concretos en función de lo que demandaban los alumnos del curso 2002-2003; se ha perfeccionado el portal web y se ha formado a los alumnos en su uso; etc.

\section{BIBLIOGRAFÍA}

ÁLVAREZ PÉREZ, P. (2002). La función tutorial en la Universidad. Una apuesta por la mejora de la calidad de la enseñanza. Madrid: EOS.

ÁLVAREZ ROJO, V. y LÁZARO MARTíNEZ, A. (2002). Calidad de las Universidades y Orientación Universitaria. Málaga: Aljibe.

BADENES LUZ, I. (1997). Proyecto de tutorización de alumnos-consejeros en la Universidad Politécnica de Valencia. AEOP Valencia. Jornada.

BENAVENT, J.A. y FOSSATI, R. (1990). Un programa de compañero-tutor para los alumnos de la Orientación Educativa de la Universidad de Valencia. Revista Española de Orientación Educativa y Vocacional, 1 (0), 66-80.

BISQUERRA ALZINA, R. (coord.) (1998). Modelos de orientación e intervención psicopedagógica. Barcelona: Praxis.

CAMPOY ARANDA, T.J. y PANTOJA VALLEJO, A. (1999). Necesidades de orientación para la mejora de la calidad de la enseñanza: un estudio descriptivo en la Universidad de Jaén. IX Congreso Nacional de Modelos de Investigación Educativa, 300-304. Málaga: AIDIPE.

CAMPOY ARANDA, T.J. y PANTOJA, A. (2000). La Orientación en la Universidad de Jaen: satisfacción/insatisfacción del alumnado y necesidades con relación al servicio. I Jornadas Andaluzas de Orientación y Psicopedagogía. Universidad de Jaén: Jaén.

CASTELLANO MORENO, F. (1994). Evolución de las necesidades de orientación educativa en universitarios de la Facultad de CC.EE. de Granada. Revista Española de Orientación Educativa y Vocacional, 57-74.

CASTELLANO MORENO, F. (1995). La orientación educativa en la Universidad de Granada. Granada: Servicio de Publicaciones de la Universidad de Granada.

CASTELLANO, F. y DELGADO, L. (1996). Un programa de orientación universitaria: Participa. Revista de Educación de la Universidad de Granada, 9, 65-78.

COROMINAS, E. e ISÚS, S. (1998). Transiciones y orientación. Revista de Investigación Educativa, 16, 155-184

DíAZ ALLUÉ, M.T. (1973). Estudio de la problemática académica del universitario madrileño. Tesis Doctoral. Madrid: Universidad Complutense.

DíAZ ALLUÉ, M.T. (1989). La Orientación universitaria ante la problemática académica y profesional. Madrid: Narcea.

DONOSO, T. y MARÍN, M.A. (1989). Las necesidades y problemática del estudiante universitario español desde la perspectiva de la orientación académica y profesional. En I Simposio sobre Orientación Académica y Profesional en la Universidad. Barcelona. 
ECHEVERRÍA SAMANES, B. (1997). Los servicios de orientación universitarios. En P. Apodaca \& C. Lobato (Eds.), Calidad en la Universidad: Orientación y evaluación. Barcelona: Laertes.

ECHEVERRÍA SAMANES, B. y RODRÍGUEZ ESPINAR, S. (1989). Las necesidades y problemática del estudiante universitario español desde la perspectiva de la orientación académica y profesional. En I Simposio sobre orientación académica y profesional en la Universida. Barcelona.

GABINETE PSICOPEDAGÓGICO (2003). Proyecto de tutoría entre compañeros (PTEC) de la Universidad de Granada. http://www.ugr.es/ ve/proyectos.html

GARCÍA JIMÉNEZ, E.; RUIZ DE MIGUEL, C.; ROMERO RODRÍGUEZ, S. y VALVERDE MACIAS, A. (2003). Innovación en la orientación universitaria: la mentoría como respuesta. Contextos Educativos. Revista de educación, 6-7, 87-112.

LÁZARO MARTÍNEZ, A. (1997): La acción tutorial de la función docente universitaria. En APODACA P. y LOBATO, C. (Eds.): Calidad en la Universidad: orientación y evaluación. Barcelona: Laertes.

LEYVA, A. y OLIVEROS, L. (1996). Una experiencia de tutoría entre iguales y de evaluación formativa. En Innovación Pedagógica y Políticas Educativas. Actas Congreso Nacional de Pedagogía, 168-170. San Sebastián: Sociedad Española de Pedagogía-Universidad País Vasco.

LOBATO, C. y MUÑOZ, M. (1994). Diagnóstico de necesidades de orientación universitaria. En XV Congreso Internacional de Recursos Humanos, Orientación.

LÓPEZ FRANCO, E. y OLIVEROS, L. (1999). La tutoría y la orientación en la Universidad. En Revista de Orientación y Psicopedagogía, 10 (17), 83-98.

MUÑOZ, M.A. y OLIVEROS, L. (1998). La orientación de recursos humanos en mercados flexibles. En REPETTO, E. y VELAZ, C.(eds), Orientación de la Carrera, recursos humanos y mercado laboral. Madrid: UNED.

PÉREZ BOULLOSA, A. (1995). El acceso de los estudiantes a la Universidad: incidencia de las elecciones en el proceso de preinscripción. En AIDIPE (Ed.) Estudios de investigación educativa en intervención psicopedagógica. Valencia.

PÉREZ BOULLOSA, A. (1998). La Orientación en la Universidad: un análisis para la reflexión. En DE SALVADOR, X.; RODICIO, M.L. (Coords). ¿Para onde camiña a Orientación?. A Coruña: Universidade da Coruña.

PÉREZ JUSTE, R. y otros (1990). Servicios de Orientación en la UNED: demandas expresadas y necesidades detectadas.Revista Iberoamericana de educación superior a distancia. 3 (1), 23-56.

RODRÍGUEZ ESPINAR, S. (coord.) (1993). Teoría y práctica de la orientación educativa. Barcelona: PPU.

ROMERO, S.; VALVERDE, A.; GARCÍA JIMENEZ, E.; SÁNCHEZ, C.; MACÍAS GUARASA, J.; GARCÍA GARCÍA, M.; GARCÍA NIETO, N.; OLIVEROS, L. y RUIZ DE MIGUEL, C. (2003). Proyecto Mentor: la mentoría en la universidad. En Investigación y Sociedad. Actas del XI Congreso Nacional de Modelos de Investigación Educativa. Granada: AIDIPE.

VALVERDE, A.; GARCÍA, E.,y ROMERO, S. (2001). La función tutorial en la Universidad de Sevilla. La mentoría como respuesta: el proyecto SI.M.U.S. Primeras Jornadas Nacionales sobre la Función Tutorial en la Universidad. La Laguna: Tenerife. (En prensa). 
VALVERDE, A.; GARCÍA, E. y ROMERO, S. (2001). Desarrollo de un sistema de alumnos mentores en la Universidad de Sevilla. Revista de Investigación Educativa, 19 (2), 626-627.

VALVERDE, A.; GARCíA, E. y ROMERO, S. (2002). Seville University's Students Mentoring Programme (SI.M.U.S.). A Guidance Experience in the Spanish University, through the training of Students Mentors. En European Conference of Educational Research. Lisboa (en prensa).

ZAMORANO, P. y OLIVEROS, L. (1998). Análisis de necesidades orientadoras en los alumnos de primer curso de la Facultad de Educación. En REPETTO, E. y VELAZ, C.(eds), Orientación de la Carrera, recursos humanos y mercado laboral. Madrid: UNED.

ZAMORANO, P. y OLIVEROS, L. (1998). Análisis de necesidades orientadoras en los alumnos de primer curso de la Facultad de Ciencias de la Educación- Centro de Formación del Profesorado. En REPETTO, E. y VELAZ, C.(eds), Orientación de la Carrera, recursos humanos y mercado laboral. Madrid: UNED. 